\title{
À CHEVAL SUR LA FRONTIÈRE ENTRE DEUX ÉTATS
}

Figure chevaline et représentations identitaire, culturelle et spatiale dans Faites de beaux rêves de Jacques Poulin

STRADDLING THE BORDER BETWEEN TWO STATES

HORSE FIGURE AND IDENTITY-BASED, CULTURAL AND SPATIAL REPRESENTATIONS IN JACQUES POULIN'S FAITES DE BEAUX RÊVES

A CABALLO EN LA FRONTERA ENTRE DOS ESTADOS FIGURA CABALLUNA Y REPRESENTACIONES IDENTITARIA, CULTURAL Y ESPACIAL EN FAITES DE BEAUX RÊVES, DE JACQUES POULIN

\section{Isabelle Proulx}

Volume 45, numéro 2 (134), hiver 2020

Jacques Poulin

URI : https://id.erudit.org/iderudit/1069509ar

DOI : https://doi.org/10.7202/1069509ar

Aller au sommaire du numéro

Éditeur(s)

Université du Québec à Montréal

ISSN

0318-9201 (imprimé)

1705-933X (numérique)

Découvrir la revue

Citer cet article

Proulx, I. (2020). À CHEVAL SUR LA FRONTIÈRE ENTRE DEUX ÉTATS : figure chevaline et représentations identitaire, culturelle et spatiale dans Faites de beaux rêves de Jacques Poulin. Voix et Images, 45(2), 33-51.

https://doi.org/10.7202/1069509ar
Résumé de l'article

Faites de beaux rêves de Jacques Poulin raconte les aventures de Limoilou, d'Amadou et de son frère Théo lors d'une joyeuse escapade au Mont-Tremblant où ils assistent au Grand Prix du Canada de Formule 1. Entre les courses des « dix mille chevaux lâchés d'un coup " sur la piste, ce trio de gais lurons passera son temps « à inventer toutes sortes d'histoires ». La mise en récit du réel et de l'imaginaire sera pour eux l'occasion d'une véritable

" métamorphose ludique de l'ego » (Ricoeur). Incarnant une sorte de Don Quichotte québécois aux allures de mousquetaire chevaleresque et de cowboy téméraire, Théo s'avèrera être à la fois un modèle et un guide pour Amadou, « commis aux écritures » en quête de son identité. Cette étrange petite fable poulinienne est en quelque sorte une invitation amicale à se laisser prendre au jeu de la littérature, autrement dit, à apprendre à rêver le monde autrement. 


\section{À CHEVAL SUR LA FRONTIÉRE ENTRE DEUX ÉTATS}

Figure chevaline et représentations identitaire, culturelle et spatiale dans Faites de beaux rêves de Jacques Poulin

$$
+++
$$

\section{ISABELLE PROULX}

Université de Sherbrooke

Les choses que tu fais, expliqua le commis, peut-être que tu pourrais pas les faire si quelqu'un avant toi y avait pas rêvé assez longtemps ${ }^{1}$.

Faites de beaux rêves de Jacques Poulin raconte la singulière histoire d'une bande d'amateurs de course automobile réunis pendant quatre jours à la piste du MontTremblant pour assister au Grand Prix du Canada de formule 1. Théo, son frère Amadou et Limoilou forment un noyau primaire auquel se grefferont, entre autres, Jane et la Ceinture verte. Le cadre que représente la course constitue un ancrage référentiel auquel s'arrime l'essentiel, soit les aventures fabuleuses des protagonistes, fabuleuses au sens de fable, puisqu'il s'agira en essence d'aventures imaginaires. "On passe notre temps à inventer toutes sortes d'histoires» (FBR, 181), dit Amadou. En toile de fond, le vrombissement des moteurs qui s'éclatent sur la piste: «Fracas insoutenable de dix mille chevaux lâchés d'un coup. Un bruit fou. Irréel.» (FBR, 187)

Roman de l'entre-deux, Faites de beaux rêves se situe entre cet avant et cet après qui nous sont si familiers: avant lui, le «roman du pays» des années 1960, contestataire et révolutionnaire pour une large part; après lui, le roman plus intimiste à survenir dans les années 1980, marqueur d'une sensibilité nouvelle caractérisée par une ouverture sur le monde, une fascination pour l'Autre ainsi qu'un refus croissant des «oppositions de naguère ${ }^{2} »$. La publication de ce quatrième récit de l'auteur survient à mi-chemin entre, d'un côté, celle de Mon cheval pour un royaume (1967) dans lequel un acte terroriste est perpétré au cœur de la ville de Québec - espace géographique et symbolique renvoyant à l'histoire de la Nouvelle-France et au souvenir de la Conquête - et, de l'autre, la publication de Volkswagen Blues (1984), «grand

1 Jacques Poulin, Faites de beaux rêves, Montréal, Bibliothèque québécoise, coll. «Littérature. BQ», 1988, p. 127. Désormais, les références à cet ouvrage seront indiquées par le sigle FBR suivi du folio, et placées entre parenthèses dans le texte.

2 Michel Biron, «Par delà les oppositions de naguère», Québec français, no 175, 2015, p. 73-75, en ligne: https://www.erudit.org/fr/revues/qf/2015-n175-qf02472/81396ac.pdf (page consultée le 8 novembre 2019). 
roman des Amériques ${ }^{3} »$ dessinant les contours d'un ailleurs lointain sur le continent nord-américain, un espace à conquérir, une liberté à s'approprier.

«Entre-deux», cette petite fable mystérieuse l'est cependant à plus d'un titre, comme l'ont souligné plusieurs commentateurs ${ }^{4}$. Dans l'introduction de l'édition de 1988 intitulée «Vivre entre le rêve et la réalité», Pierre Filion écrivait que "ces personnages inventent, jouent des personnages eux-mêmes à l'intérieur de leur propre errance, se construisent un lieu auquel ils finissent par croire, parce que la parole peut faire tout exister et tout disparaître, et qu'ainsi tout est possible» (FBR, 6-7). La lecture que nous proposons du roman se veut un approfondissement de cette question de l'entre-deux, espace polarisé entre la réalité et le rêve, et plus particulièrement des conséquences qu'entraîne la perméabilité du réel à l'imaginaire dans le processus de transformation identitaire des protagonistes ainsi que de l'impact qu'elle peut avoir sur leur rapport à la culture et à l'espace.

3 «Après la crise d'Octobre, le roman se fait plus intime. Plusieurs héros [...] arpentent le territoire nordaméricain à la recherche des vastes espaces, attirés, comme leur ancêtre Jack Kerouac, par le mythe américain [...]. Pensons à [...] Jacques Poulin et à son Volkswagen Blues (1984), le grand roman des Amériques, comme je me plais à l'appeler [...].» Aurélien Boivin, «La littérature québécoise: une littérature de l'Amérique», Québec français, n 154 , été 2009, p. 62; nous soulignons.

4 Dans une perspective de sociologie de la littérature, François Gallays met en exergue le rôle de médiation qu'est appelé à jouer le récit entre la production et la réception du bien symbolique: «Comme il n'est jamais donné au lecteur d'assister à une course autrement que par des voies indirectes, le discours du roman semble s'interposer à la façon d'un médiateur entre le lecteur et le savoir codé des courses automobiles.» (François Gallays, "Faites de beaux rêves», Maurice Lemire [dir.], Dictionnaire des œuvres littéraires du Québec, t. V: 1970-1975, Montréal, Fides, 1987, p. 332, en ligne: http://services.banq.qc.ca/sdx/DOLQ/ document.xsp?id=04225\&cv=04\&qid=sdx_q1 [page consultée le 8 novembre 2019].) Deux autres couples oppositionnels sont révélés par Jaap Lintvelt (Jaap Lintvelt, Aspects de la narration: thématique, idéologie et identité. Guy de Maupassant, Julien Green, Anne Hébert, Jacques Poulin, Québec/Paris, Nota bene/ L'Harmattan, coll. «Littérature[s]», 2000, 306 p.). Son examen des huit premiers romans de Jacques Poulin mène à la découverte d'une «fusion harmonieuse» (p. 218) des oppositions liées à la problématique identitaire alors que le couple homme/femme s'abolit pour faire place à la figure de l'androgyne, tandis que du doublet américanité/francité naît la québécité. Enfin, on trouve au sein de l'œuvre les traits oppositionnels modernité/postmodernité. À ce chapitre, Faites de beaux rêves semble être une œuvre limitrophe si l'on en croit la critique. Parlant de la production romanesque de Poulin jusqu'à Volkswagen Blues, Gilles Marcotte écrit: «Cette œuvre d'apparence si légère se révèle hantée, traversée, par la problématique essentielle de la modernité. Par sa forme, plus encore que par ses thèmes, elle dit que la parole, l'écriture, comportent les risques les plus graves parce qu'elles ne reposent plus sur aucune convention, aucune tradition.» (Gilles Marcotte cité dans Laurent Mailhot, "Présentation. Le voyage total», Études françaises, vol. XXI, n 3 , hiver 1985, p. 4, en ligne: https://www.erudit.org/fr/revues/etudfr/1985-v21-n3-etudfr1632/036864ar. pdf [page consultée le 8 novembre 2019].) Dans un article intitulê «Être ou ne pas être postmoderne au Québec» (1995), André Lamontagne fait pour sa part état de la "présence d'un corpus plus résolument postmoderne» dans lequel il range, entre autres, «les quatre ou cinq derniers romans de Jacques Poulin»; un rapide décompte permet de constater que le cinquième de ces romans n'est nul autre que Faites de beaux rêves. (André Lamontagne, "Être ou ne pas être postmoderne au Québec», Liberté, vol. XXXVII, n 4 , août 1995, p. 42, en ligne: http://id.erudit.org/iderudit/32321ac [page consultée le 8 novembre 2019].) Enfin, soulignant avec Ginette Michaud l'inachèvement et l'incomplétude qui caractérisent la prose d'Amadou, Pierre Hêbert soutient quant à lui qu'« [i]l s'agit là d'un trait important des récits postmodernes: une fin ouverte, suspendue, des images hybrides. Dès lors, poursuit-il, [...] les deux métarécits d'Amadou concernant le hockey et le bureau, par leur refus de conclure, représentent probablement les premières caractéristiques du postmoderne chez Poulin, lequel ressortira davantage dans Les grandes marées et Volkswagen Blues [...] ». Pierre Hébert, Jacques Poulin. La création d'un espace amoureux, Ottawa, Presses de l'Université d'Ottawa, coll. «Euvres et auteurs», 1997, p. 103-104. 
Une traversée de l'univers fantasmagorique de Faites de beaux rêves nous permettra de découvrir qu'Amadou vit une aventure dans laquelle se confondent la quête identitaire et la quête de l'imaginaire. D'entrée de jeu, cet écrivain en devenir se trouve dans un état larvé, emmailloté qu'il est dans le "sac familial» (FBR, 198), véritable chrysalide de laquelle il sortira papillon un jour prochain. Sur le plan symbolique, ce personnage incarne le récit hégémonique du discours culturel québécois 5 . En opposition à ce que l'on observe chez le commis aux écritures («nous» centrifuge, espace clos, temps figé de la mémoire refoulée, action empêchée), on trouve chez Théo un ensemble de traits qui, par leur seule présence au sein du roman, permettent d'ébranler dans ses fondements le noyau du discours culturel, un récit dominant, admis et célébré comme allant de soi. Sous les apparences d'un homme à cheval (au cheval invisible!) dont les multiples visages travaillent le texte d'une manière dialogique, en synergie avec les procédés de carnavalisation, se trouve un être polyglotte (québécois populaire, français normatif et littéraire, anglais, allemand) au bagage culturel hétérogène (culture européenne/américaine, savante/populaire), chevauchant par tous les chemins, horizontalement (France/Belgique/Espagne/Italie) et verticalement (États-Unis/Mexique/Amérique du Sud). Sorte de Don Quichotte québécois en quête d'aventures réelles et imaginaires, réalisant aussi la synthèse du mousquetaire français chevaleresque et du téméraire cowboy américain, Théo se présente comme la «fusion harmonieuse ${ }^{6}$ » de toutes les oppositions qui le définissent, contrairement à son "vieux frère» Amadou, image de la division du soi et de l'inadéquation au monde.

Après avoir tracé un bref portrait des deux principaux personnages de l'histoire et circonscrit les enjeux de leur parcours commun, nous ancrerons notre problématique dans un socle théorique d'inspiration sociocritique fondé notamment sur la thèse avancée par Micheline Cambron dans Une société, un récit et sur les réflexions d'André Belleau à propos du carnavalesque bakhtinien. Partant de là, nous verrons comment Théo, personnage associé à la figure du cheval, joue un rôle essentiel dans la quête d'Amadou, ce qui nous permettra de tirer des conclusions sur le rôle de l'imaginaire dans le cheminement des êtres de papier et, plus largement, dans la transformation de la société québécoise.

\section{DEUX PERSONNAGES, UNE PROBLÉMATIQUE}

Faites de beaux rêves met en scène deux frères - Amadou et Théo, plus jeune de deux ans $(F B R, 61)$ - qui ont grandi ensemble dans le «Vieux-Québec» $(F B R, 76)$. Passionné de course automobile, le cadet voyage dans divers pays pour assister aux courses de formule 1. Il gagne sa vie à "préparer, pour les revues de sport automobile, des reportages aussi étoffés et vivants que possible» $(F B R, 22)$. Théo ne s'en

5 Micheline Cambron, Une société, un récit. Discours culturel au Québec (1967-1976), Montréal, l'Hexagone, coll. «Essais littéraires», 1989, p. 176.

6 Jaap Lintvelt, Aspects de la narration. Thématique, idéologie et identité, Québec, Éditions Nota Bene/Paris, L'Harmattan, 2000, p. 218. 
cache pas: «Je ne suis pas un vrai journaliste. L'argent des reportages, c'est pour ma carrière de pilote en Europe. » $(F B R, 26)$ Cependant, rien ne semble plus douteux que cette carrière de «pilote de Formule 3 » $(F B R, 26,195)$. Ainsi, derrière le « célèbre pilote» $(F B R, 26)$ se cache un très bon acteur, un sacré farceur. C'est d'ailleurs lui qui occupera l'avant-scène tout au long du récit. Toutefois, le personnage principal est Amadou. Il incarne la figure de l'écrivain, centrale dans l'œuvre de Jacques Poulin, comme le souligne Pierre Filion: «Amadou, le commis aux écritures, présent dans tous les romans de Poulin, déguisé sous des noms différents et des professions diverses, mais toujours pivot central de l'émotion et de la focalisation. » $(F B R, 6) \mathrm{Ce}$ commis aux écritures (désignation qui n'apparaît que dans Faites de beaux rêves), Jean-Pierre Lapointe le décrit comme un être «solitaire, mélancolique et passif, dont le passé est hanté par le souvenir" » et "la quête pourtant si méticuleuse de l'excellence au travail et au jeu aboutit à un constat d'insignifiance et d'échec ${ }^{8}$ ». En contrepartie, Pierre Hébert présente le jeune esthète sous un jour avantageux: «Théo et Limoilou sont des transcripteurs, le premier, de la réalité, la seconde, de la tradition. L'entreprise d'Amadou est autrement plus périlleuse, car il est "le seul à véritablement inventer des histoires, à prendre le risque de l'invention" ${ }^{9}$. » Il n'en demeure pas moins que le protagoniste croit peu en son talent - «ça peut être plate à mort» $(F B R, 81)$, dit-il - et qu'il ne se reconnaît pas un statut d'écrivain ${ }^{10}$ : «- Je ne suis pas écrivain. - Tu es quoi ? - Commis aux écritures.» (FBR, 196) La sémantique du titre qu'il s'attribue témoigne de la dualité fondamentale d'un personnage se situant entre deux états identitaires conflictuels. D’une part, «commis» désigne un «agent subalterne» (Le Petit Robert) dans le cadre d'une structure administrative hiérarchique. D'autre part, le mot «écritures» renvoie à l'idée d'une parole sacrée (Les Écritures, la Bible) et signale en quelque sorte la révélation d'une Vérité profonde. En clair, ce titre de «commis aux écritures» symbolise la dissonance, l'incohésion profonde qui caractérise Amadou. Ce dernier n'est pas encore là où il voudrait être. L'espace idéal qu'il souhaite habiter est la littérature, véritable «maison de l'être ${ }^{11} »$, dans le but d'en arriver, comme le souligne Pierre Hébert, à créer «par le langage, dans le langage, le monde des significations ${ }^{12} »$. La quête d'Amadou qui s'amorce dans Faites de beaux rêves consistera à trouver des moyens de faire échec à cette désignation bipartite inconfortable - commis (aliénationempêchement-inertie)/écriture (libération-épanouissement-mouvement) - pour éventuellement la remplacer par une désignation autre à l'image d'une nouvelle

7 Jean-Pierre Lapointe, «Narcisse travesti. L'altérité des sexes chez trois romanciers québécois contemporains», Voix et Images, vol. XVIII, n 1, automne 1992, p. 14, en ligne: http://id.erudit.org/iderudit/ 200994ar (page consultée le 8 novembre 2019).

8 Ibid., p. 15.

9 Pierre Hébert, Jacques Poulin. La création d'un espace amoureux, p. 99. La citation est de Gilles Marcotte, «Histoires de zouaves», Études françaises, vol. XXI, n 3, hiver 1985, p. 11.

10 S'il se reconnaît le titre d'écrivain, c'est sous le couvert de l'ironie, de l'autodérision: « - Êtes-vous un écrivain connu? dit-elle en s'adressant au commis. - Très connu, dit Amadou: ma tante Marie, mon oncle Louis, ma cousine Hélène, mon oncle André, ma tante Albertine, mon oncle Édouard, mon cousin Antoine, ma...» (FBR, 74-75)

11 «Le langage est la maison de l'être», dit Théo traduisant la célèbre pensée de Heidegger (FBR, 93).

12 Pierre Hébert, Jacques Poulin. La création d'un espace amoureux, p. 103; l'auteur souligne. 
identité, celle d'écrivain, synonyme de "pro-création ${ }^{13}$ » esthétique du soi par le langage.

Tous les commentateurs de l'œuvre ont souligné le rôle d'adjuvant que Théo jouera dans la quête de l'imaginaire d'Amadou, indissociable de sa quête identitaire. À ce sujet, Jean-Pierre Lapointe écrit:

Le seul guide masculin qui exerce une influence certaine sur le protagoniste sera son frère aîné $[s i c]^{14}$ Théo, présence dominante dans Faites de beaux rêves, transfiguré en projection mythique dans les deux romans suivants. Autant le frère cadet [sic] est effacé, timoré, casanier, autant Théo est fougueux, audacieux, aventurier. Il a été dans l'enfance l'initiateur aux mystères du monde et conserve dans l'âge adulte, même éloigné par le temps et l'espace, un rôle de protecteur ${ }^{15}$.

Le récit est truffé de passages où Théo offre de l'aide à son frère, l'encourage, le réconforte. Cependant, plus encore que ses paroles bienveillantes et son écoute active, c'est la nature même du personnage, foncièrement duelle, qui sera la plus grande source d'inspiration pour Amadou. Mais avant de prendre la pleine mesure de cette influence bénéfique, encore faut-il cerner l'essence profonde des deux entités figuratives antinomiques que sont Amadou et Théo, ce que la notion de discours culturel nous permet de réaliser.

\section{LE DISCOURS CULTUREL QUÉBÉCOIS DANS TOUS SES ÉTATS}

Publié en 1974, le roman de Poulin se situe dans la période concernée par l'étude de Micheline Cambron. Dans Une société, un récit, cette dernière analyse le discours culturel québécois caractéristique des années 1967 à 1976 par le biais de l'étude de quatre paradigmes (sujet, action, espace, temps) au sein de productions culturelles représentatives de l'époque. Selon elle, ce discours culturel est constitué d'un «récit hégémonique» définissant en quelque sorte l'«identité narrative» de la collectivité québécoise ${ }^{16}$ et de "mécanismes de mise à distance qui, inscrits dans la lettre des textes, composent au récit hégémonique une altérité fondatrice du sens ${ }^{17}$ ». Postulant que Faites de beaux rêves est représentatif du discours culturel québécois, il devrait être possible d'y retrouver ces deux éléments constitutifs. Précisons d'emblée ce à

13 Ibid., p. 104.

14 Le critique fait erreur ici en affirmant que Théo est l'aîné et qu'Amadou est le cadet. Le passage suivant démontre sans équivoque qu'Amadou est plus vieux que Théo : «J'avais neuf ans et Madou onze.» $(F B R, 61)$

15 Jean-Pierre Lapointe, «Narcisse travesti. L'altérité des sexes chez trois romanciers québécois contemporains», p. 15.

16 «C'est donc dans un rapport au temps, à l'Histoire et ultimement à sa propre identité, qui se révèle articulée sur un mode paradoxal, que se donne à lire le récit commun du discours culturel québécois des années 1967-1976. Ce récit commun raconte la radicale perplexité de la collectivité québécoise face à la définition hégémonique de son identité, qui sert de lieu d'ancrage dans la longue durée à son désir d'évolution dans la courte durée.» Micheline Cambron, Une société, un récit. Discours culturel au Québec (19671976), p. 180.

17 Ibid., p. 177. 
quoi ils réfèrent. D'une part, le récit hégémonique propre au discours culturel commun aurait la particularité d'être transhistorique, c'est-à-dire qu'il serait demeuré fidèle à lui-même depuis le xix ${ }^{e}$ siècle jusqu'à nos jours ${ }^{18}$. En voici les paramètres:

Le sujet réfléchissant y est toujours un «nous» englobant et monolithique, qui est défini circulairement par l'espace clos qui le circonscrit. La conception du temps repose sur une valorisation implicite du passé et sur une éradication du futur qui font du présent un temps de continuation, de permanence. L'espace est clos, il se ramène à des lieux de proximité et d'appartenance, et aucun mouvement ne semble nécessaire à sa construction conceptuelle. La logique des actions, enfin, étant contrainte par un temps et un espace sans perspectives et enfermée dans l'organisation spéculaire d'un «nous» immobile, exclut toute transformation ${ }^{19}$.

En opposition à ce «noyau dur remplissant des fonctions hégémoniques» se dressent différentes stratégies discursives permettant de le relativiser, voire de le critiquer, «des procédés de caractère générique, lesquels expriment la manière privilégiée dont chaque genre littéraire établit une distance à l'égard du réel tel que la doxa du récit hégémonique le véhicule ${ }^{20} »$ : ironie, tragique, lyrisme, etc. Dans le cas du roman, le dialogisme tient souvent lieu de procédé de distanciation. Il se définit comme «l'existence et la concurrence de plusieurs "voix" dans un texte où s'expriment des points de vue idéologiques ou sociaux divergents, voire incompatibles ${ }^{21} »$. L'un des supports privilégiés de ces «voix» concurrentes est le personnage. Comme le souligne André Belleau, «les mots ne sont pas là pour constituer des personnages ainsi que le croit une sémiotique naïve, au contraire les personnages existent précisément pour que les mots soient prononcés (Gary Saul Morson) ${ }^{22} »$. Dans cette perspective, Amadou et Théo doivent être envisagés comme des "phénomènes textuels" produits par les interactions dialogiques des énoncés. Par ailleurs, il importe de signaler que cette opposition littéraire à la doxa du discours social ne se fait pas toujours sans douleur, comme le constate Fernand Dumont:

Par l'abolition momentanée des rapports de la conscience et du monde, une autre conscience et un autre monde peuvent surgir à qui il suffira de devenir poème, tableau ou roman pour qu'ils s'opposent, et pour toujours, aux bruits familiers de la conscience mondaine, leur troublant et énigmatique défi, leur inguérissable blessure ${ }^{23}$.

18 Ou du moins jusqu'à 1989, date de publication de l'étude de Micheline Cambron.

19 Ibid., p. 175-176.

20 Ibid., p. 178.

21 Constanze Baethge, «Dialogisme», Paul Aron, Denis Saint-Jacques et Alain Viala (dir.), Le dictionnaire du littéraire, avec la participation à la direction scientifique de l'ouvrage de Marie-Andrée Beaudet, $3^{e}$ édition augmentée et actualisée, Paris, Presses universitaires de France, coll. «Quadrige. Dicos poche», 2010, p. 181.

22 André Belleau, «La dimension carnavalesque du roman québécois», Jacques Pelletier, Littérature et société. Anthologie, avec la collaboration de Jean-François Chassay et de Lucie Robert, Montréal, VLB éditeur, coll. «Essais critiques», 1994, p. 224-225.

23 Fernand Dumont cité dans Ibid., p. 183-184. 
Caractéristique du discours culturel québécois, «cette blessure» demeure selon Cambron la source d'une souffrance irréductible ${ }^{24}$. Dans son ouvrage Un coin dans la mémoire, Yvan Lamonde évoque sensiblement la même problématique. Selon lui, les stratégies colonisatrices britanniques mises en place au cours de l'histoire, fondées sur le principe "diviser pour régner ${ }^{25}$ ", auraient eu pour effet de créer un sentiment d'incertitude, d'indécision généralisée dans la société québécoise ${ }^{26}$, comme le révèle un certain nombre d'affects criants (sentiment de pauvreté, de fatigue culturelle, d'inachèvement, d'ambivalence, etc.), créant du coup une fissure dans la mémoire de la collectivité. Plutôt que d'envisager la Conquête comme un "trauma», comme d'autres avant lui, Lamonde préfère s'attarder à l'avant et à l'après de ce nœud de l'histoire nationale. Ainsi, écrit-il, «il y a un [...] moment d'avant la division, un moment d'unité de soi. Un paradis perdu sur lequel la mémoire s'est figée ${ }^{27}$ ». La conséquence d'un tel rapport déficitaire au passé peut engendrer selon lui une attitude amnésique volontaire, cela afin de colmater la brèche et de calmer le mal ${ }^{28}$.

Ce cadre théorique nous permet de mieux comprendre la dichotomie des «voix» concurrentes que représentent au sein de la fiction les personnages des deux frères. Du point de vue de la théorie de Cambron, Amadou incarnerait le récit hégémonique. Pendant le séjour à Mont-Tremblant, ce dernier préfère la compagnie du noyau des intimes (Limoilou et Théo), aime s'emmailloter avec eux dans le «sac de couchage familial» (FBR, 153, 156-157), ne participe pas aux jeux organisés avec les festivaliers, ne parvient pas à nouer des relations intimes avec les filles de l'extérieur. Sur le plan de la création littéraire, on remarque sa difficulté à amorcer ses récits (FBR, 81-83), lesquels resteront également inachevés ( $F B R, 54,92)$, signe de la faible valeur transformatrice de ses actions. Par ailleurs, le rapport d'Amadou au temps est problématique: il ressasse des souvenirs d'enfance (FBR, 128), s'attarde plutôt au passé qu'à l'avenir (FBR, 123, 128-130, 199-200). Sur le plan spatial, la piste de course symbolise le lieu clos. Certes, il y a mouvement, mais celui-ci demeure circulaire. Une force centrifuge semble empêcher le sujet Amadou de prendre son élan dans le monde, à l'extérieur du cercle d'appartenance. La preuve en est qu'après la course, il décline l'invitation à se joindre au groupe en partance vers une nouvelle destination. Il choisit plutôt de se réfugier dans le cocon du «nous» hégémonique afin de s'évader dans le sommeil. S'inclinant respectueusement devant le choix de son frère, Théo dit: "Un homme peut pas trouver de meilleure place pour dormir que la vieille Gipsy. Les ressorts sont mous et t'as l'impression que ta mère te berce dans ses bras pour t'endormir, je te mens pas.» (FBR, 199-200) Nu dans le grand sac de couchage (FBR, 200-201), ayant avalé «trois pilules avec une gorgée de bière» $(F B R, 198)$, Amadou se prépare à dormir «vingt-quatre heures» $(F B R, 198)$. Ainsi

\footnotetext{
24 Ibid., p. 184.

25 Yvan Lamonde, Un coin dans la mémoire. L’hiver de notre mécontentement, Montréal, Leméac, coll. «Phares», 2017, p. 15.

26 Ibid., p. 104.

27 Ibid., p. 105

28 «Toute situation qui met en cause cette représentation de soi comme intouchable comporte le risque de voir refouler le refoulé. Comporte la douleur de devoir revivre la perte première, primale, de l'unité. La fidélité à soi-même serait cette fidélité-là, cette fidélité à un intouchable [...].» Ibid.
} 
s'enfoncera-t-il dans un lourd sommeil artificiel et paralysant, le corps bercé dans les bras de sa "mère», la conscience étouffée par l'ingestion d'un mélange d'alcool et de somnifères puissants. Nul doute: Amadou est un être souffrant qui se retrouvera au réveil «seul comme un chien» $(F B R, 10)$. Sur le plan symbolique, il incarne bien cette douloureuse «blessure» dont parlait Cambron à la suite de Dumont, et cette blessure paraît pour le moment inguérissable.

Pour sa part, le personnage de Théo apparaît comme une "construction hybride complexe ${ }^{29}$ " favorisant la distanciation par rapport à la doxa du discours culturel. Tout d'abord, le rapport au temps chez Théo est autre que celui d'Amadou. Aucunement nostalgique du passé (FBR, 128-130), il jouit pleinement du présent tout en se projetant dans l'avenir (FBR, 130). Constatant que, pour les pilotes, «le temps s'élargit» (FBR, 157), Théo fait lui aussi l'expérience d'un autre espace-temps, celui de l'Histoire. Par ses jeux de rôle, il met à distance le récit commun de manière à le transcender. La théâtralisation lui permet en quelque sorte de réinventer le parcours de sa collectivité: "On a organisé la plus grande partie de fresques historiques depuis la Confédération. [...] [T]out le monde s'est vengé des maudits Anglais. C'est pour ça que le vieux Théo est magané à mort [...].» $(F B R, 149)$ Par ailleurs, il fréquente avec plaisir des gens hors du cercle restreint de la famille. Constamment en action, toujours lucide malgré son penchant pour l'alcool (FBR, 37), Théo n'hésite pas à sortir du lieu «national», comme en témoigne son départ pour les États-Unis à la fin du récit, dans une sorte de "[m]ouvement de réappropriation symbolique du continent américain ${ }^{30} »$. Bref, envers et contre le récit hégémonique, s'étant lesté d'une part encombrante de son passé (personnel/collectif) pour ne conserver que «le soi, plein de l'antérieur métabolisé ${ }^{31}$ », Théo semble avoir réalisé le travail de deuil menant selon Lamonde «à un être souverain, à un acte souverain capable de créer un événement qui ouvre sur une nouvelle histoire ${ }^{32} »$. Et c'est sur ce mot histoire qu'il faut insister, car s'il est une chose que l'on doit retenir par rapport à l'être et au faire de Théo, c'est cette propension à jouer à être un autre que soi-même tout en demeurant lui-même. Comme l'écrit Paul Ricœur, «[l]a métamorphose du monde, selon le jeu, est aussi la métamorphose ludique de l'ego ${ }^{33}$ ». Parmi le groupe, Théo est celui qui jouera le plus grand nombre de rôles, lesquels sont autant de "variations imaginatives de l'ego ${ }^{34} »$. En somme, il est l'incarnation exemplaire du double identitaire, et $c^{\prime}$ est à cet aspect du personnage que nous nous attarderons maintenant en étudiant la figure du cheval, à laquelle il est intrinsèquement lié.

29 Mikhaïl Bakhtine cité dans André Belleau, «La dimension carnavalesque du roman québécois», p. 221.

30 Jean Morency, «L'errance dans le roman québécois», Québec français, n 97, printemps 1995, p. 82, en ligne: https://www.erudit.org/fr/revues/qf/1995-n97-qf1229595/44320ac/ (page consultée le 8 novembre 2019).

31 Yvan Lamonde, Un coin dans la mémoire. L'hiver de notre mécontentement, p. 106.

32 Ibid., p. 107.

33 Paul Ricœur cité dans Olivier Abel et Jérôme Porée, Le vocabulaire de Paul Ricœur, Paris, Ellipses, coll. «Vocabulaire de...», 2009, p. 52; l'auteur souligne.

Ibid. 


\section{LE CHEVAL, MOTEUR DE L'ACTION}

«L'art de dire, chez Poulin, c'est toujours de ne pas dire directement» (FBR, 7), écrit Pierre Filion dans son introduction au roman. Ginette Michaud abonde dans le même sens. Selon elle, «les détails deviennent ici de véritables opérateurs textuels, d'autant plus efficaces qu'ils servent à faire voir tout en se dérobant eux-mêmes à la vue ${ }^{35}$ ". Au début du premier chapitre de Faites de beaux rêves apparaît cette phrase banale: «Théo mesurait six pieds et deux. Il était vêtu d'un short en coutil et il portait son fameux chapeau de Camargue.» $(F B R, 13)$ Au fil des pages, nous remarquons la fréquente apparition du chapeau ${ }^{36}$. En tout, l'œuvre de 200 pages comporte 31 occurrences de l'expression "chapeau de Camargue»; elle se trouve dans l'incipit et l'excipit, et apparaît dans les quatre parties du récit selon une distribution homogène. Un martèlement à ce point soutenu d'un détail vestimentaire interdit d'y voir un simple «effet de réel» visant à assurer l'illusion référentielle. Ainsi, du «petit-détail-quifait-vrai ${ }^{37}$ » qu'il était au départ, ce chapeau prend valeur d'indice, de signe ${ }^{38}$. Théo serait-il un homme à cheval? Le cas échéant, où se cache sa monture ${ }^{39}$ ? Puisque nous avons affaire chez cet auteur à «une écriture qui cherche bien plus à suggérer qu'à expliquer ${ }^{40}$ ", comme le souligne Jean Morency, il ne reste plus qu'à observer à la loupe les chevaux qui transparaissent en filigrane.

En 1992, Jean-Pierre Lapointe proposait une typologie des figures de la masculinité dans l'œuvre de Jacques Poulin. Selon lui, «l'anti-héros marginal [le personnage écrivain] se définit lui-même par une comparaison déficitaire à des modèles masculins stéréotypés ${ }^{41} »$. Les trois types de modèles servant à la comparaison sont l'homme ordinaire, le héros légendaire et le guide ou le mentor. Dans Faites de beaux rêves, c'est à «Théo le héros» et à «Théo guide et mentor» que s'identifiera

35 Ginette Michaud, «Récits postmodernes?», Études françaises, vol. XXI, n³ 3, hiver 1985, p. 77, en ligne: http://id.erudit.org/iderudit/036870ar (page consultée le 8 novembre 2019).

36 Il semble que Théo se soit procuré ce chapeau lors d'un séjour aux «Saintes-Maries-de-la-Mer» (FBR, 199), capitale de la Camargue (France).

37 Ginette Michaud, «Récits postmodernes?», p. 75

38 «[O]n entrevoit ce qu'une lecture détaillée de l'infiniment petit peut donner lorsque le détail cesse d'être le simple véhicule de données sociologiques ou lorsque, détotalisé, il ne renvoie plus à l'ensemble du texte ou au monde comme macrocosme. Dans les textes de Poulin, le détail force le lecteur à constamment revenir sur ses opérations de lecture et lui enjoint, entre autres, de réinterpréter en retour tout le contexte (Poulin affirmera à plusieurs reprises l'importance de la relecture: pas de lecture sans relecture). Ce n'est donc jamais une perte de temps que d'analyser en détail les fictions déjà si économes, si sobres et précises. Poulin n'a de cesse de nous le rappeler, à nous lecteurs trop rapides: il ne faut pas négliger ou mépriser les petits problèmes techniques puisque ce sont eux qui, le plus souvent, sont susceptibles de nous mener le plus loin.»Ibid., p. 79.

39 Hormis les «dix mille chevaux lâchés d'un coup» (FBR, 187) renvoyant métaphoriquement au bruit des moteurs des véhicules de course, l'évocation par Amadou de «Jeanne d'Arc sur son cheval» (FBR, 95) et de «trois paires de chevaux» $(F B R, 141)$ tirant un chariot dans l'un de ses rêves, il n'y a pas d'équidés à proprement parler dans le roman.

40 Jean Morency, «Le vieux Chagrin», Aurélien Boivin (dir.), Dictionnaire des œuvres littéraires du Québec, t. VIII : 1986-1990, Montréal, Fides, 2011, p. 951.

41 Jean-Pierre Lapointe, «Narcisse travesti. L'altérité des sexes chez trois romanciers québécois contemporains", p. 15. 
Amadou $^{42}$. Ces figures duelles témoignent de la carnavalisation du personnage, procédé que l'on trouve à l'œuvre dans le roman et qui, conjointement à la sémiotique du personnage, contribue à en libérer la "plurivocalité ${ }^{43}$ ». Selon André Belleau, la textualisation du carnaval procède de deux manières: par la représentation du carnaval lui-même et par le recours à des isotopies carnavalesques. Dans le récit, on trouve d'abord la représentation du carnaval ${ }^{44}$ sous la forme de fresques théâtrales, de beuveries collectives, etc., ce qu'avait déjà remarqué François Gallays ${ }^{45}$. Le carnavalesque s'y exprime également par le biais d'isotopies porteuses des caractéristiques profondément dialogiques du carnaval: oppositions, rabaissements et renversements se trouvent partout associés au burlesque Théo, incarnation parfaite du double identitaire (reporter dans la réalité et cavalier dans l'imaginaire).

\section{LE GARDIAN}

Tout au long de l'histoire, Théo porte son chapeau de Camargue qui évoque la figure du gardian à cheval, un "pasteur cavalier chargé de la surveillance et de la conduite de troupeaux de bovins et de chevaux élevés en semi-liberté dans les zones incultes du delta du Rhône ${ }^{46} »$. Ce métier apparu au Moyen Âge s'est perpétué jusqu'au début du $\mathrm{xx}^{\mathrm{e}}$ siècle. Par son rôle de protecteur de la horde, le gardian peut être associé au type du guide ou du mentor que Lapointe définit comme le «[p]rofesseur d'un savoir [...] en mesure de transcender les circonstances et d'ouvrir au protagoniste la voie de la connaissance et du dépassement de $\operatorname{soi}^{47}{ }^{4}$. Dans Faites de beaux rêves, ce "professeur» n'est nul autre que Théo, qui interprétera divers rôles: pédagogue, passeur culturel, exégète et mentor ${ }^{48}$. Le rapport qu'entretient le personnage de Théo

42 Pour ce qui est de «l'homme ordinaire» dans Faites de beaux rêves, il s'agit de «l'homme à la Kawasaki» $(F B R, 45)$, que Théo surnomme «Matousalem» (FBR, 66).

43 André Belleau, «La dimension carnavalesque du roman québécois», p. 228.

44 «- . v viens juste de finir ma Molson, dit Théo. Je ferme ma gueule parce que les couleurs des voitures, les pneus larges, l'odeur de l'huile, je trouve que c'est beau et que ça ressemble à rien d'autre, même pas au cirque Barnum \& Bailey [...].» (FBR, 183)

45 Jacques Poulin, selon François Gallays, «subvertit complètement le rituel [de la course automobile] en exhibant le caractère profondément carnavalesque des quatre journées pendant lesquelles les bières "Dow" ou "Molson", créatrices d'euphorie, rivalisent avec les "Lotus" et les "Ferrari" ». François Gallays, "Faites de beaux rêves".

46 Frédéric Raynaud, «Les gardians de Camargue et leurs chevaux, patrimoine archivistique et photographique pour une étude historique», In Situ. Revue des patrimoines, n² 27, 2015, en ligne: http://insitu. revues.org/12105 (page consultée le 8 novembre 2019).

47 Jean-Pierre Lapointe, «Narcisse travesti. L'altérité des sexes chez trois romanciers québécois contemporains», p. 15.

48 Dans le rôle du pédagogue, ce Théo «au ton distrait d'un vieux prof de littérature» ( $F B R, 151)$ offre volontiers en partage ses connaissances sur une foule de sujets, en particulier sur l'univers de la course automobile qui le passionne - Théo est une "véritable encyclopédie de la course automobile», selon Pierre Filion («Vivre entre le rêve et la réalité» $[F B R, 6]$ ). Pour Pierre Hébert aussi, « [l] grand connaisseur des courses, c'est Théo; il lui revient toujours de livrer des renseignements sur les pilotes, de raconter des exploits passés, de chercher le sens de ce jeu» (Pierre Hébert, Jacques Poulin. La création d'un espace amoureux, p. 93). Il s'agit d'un «savoir codé» recelant une certaine sagesse, selon François Gallays (François Gallays, «Faites de beaux rêves»). À maintes reprises, Théo donnera à ses comparses des leçons de vie inspirées des 
avec le gardian de Camargue est doublement intéressant si l'on considère la récente transformation de la profession:

À partir du début du xIXe siècle et à l'occasion des fêtes patronales ou nationales, les municipalités organisèrent le programme des réjouissances autour de jeux taurins. [...] [D]e simple ouvrier agricole, solitaire et un peu sauvage, sous l'Ancien Régime, il devint l'acteur indispensable des fêtes populaires ${ }^{49}$.

Ainsi, dans son rôle moderne, le gardian prend part au carnaval tout comme Théo qui, parallèlement à son rôle de guide et de mentor, agira à titre de personnage carnavalisé porteur du couple oppositionnel sérieux/zouave en constante interaction dialogique. En effet, plusieurs passages donnent à voir un Théo grave et austère (FBR, 30, 151), côté cérébral auquel s'oppose son attitude de «zouave» (FBR, 15, 36, $82)$ et sa douce folie $(F B R, 24,158)$ : rabaissement profanateur (il récite de la poésie après avoir uriné $[F B R, 87])$, travestissement (il se déguise en secrétaire, soutiengorge et jupe à l'appui [FBR, 87-88]), opposition vie/mort (aux décès tragiques des pilotes s'oppose la mort joyeuse d'un Théo débordant de vie qui craint à tout instant de tomber «raide mort de soif» ou qui est «magané à mort» d'avoir trop bu [FBR, 15, 149]), etc.

\section{PREUX CHEVALIER, CHEVALIER ERRANT}

Dans la typologie de la masculinité chez Jacques Poulin, outre la figure du guide ou du mentor se trouve celle du héros légendaire, que Lapointe définit comme «modèle normatif de l'énergie mâle dont les prouesses physiques déjouent le destin commun de la médiocrité et défient la mortalité ${ }^{50} »$. Dans le récit, ce type du héros renvoie à deux figures: le chevalier et le cowboy. Voyons d'abord celle du chevalier, qui se

expériences de coureurs de renom: «Pour piloter, dit-il citant Nuvolari, vous avez besoin de bons yeux, de bons réflexes, d'une coordination instantanée des bras et des jambes, mais, pour gagner une course automobile, il faut conduire avec son cœur.» (FBR, 154-155) De plus, c'est à lui que revient la tâche de révéler la présence signifiante et incontournable de textes sacralisés: "Le succès est quelque chose de personnel et diffère parfois totalement de la victoire." [Stirling Moss] C'est écrit dans la vieille Gipsy.» (FBR, 156) En tant que passeur culturel, Théo communique «tout ce qui lui pass[e] par la tête» $(F B R, 64)$ : littérature (Rutebeuf, du Bellay, Ronsard, Racine), philosophie (Bachelard, Heidegger), histoire (Jacques Cartier, Pearl Harbor, la Confédération, la conquête de l'Ouest, La Palice...). Par ailleurs, Théo est à l'origine du troisième récit mythique raconté par Amadou; il s'agit d'«[u]ne sorte de songe, comme [en faisaient] les Anciens» $(F B R, 144)$, attribué à Jacques Cartier au sujet de l'éventuelle mort du «Grand Rêve de l'Amérique» (FBR, 144). Enfin, notons qu'en tant que mentor, Théo aide le mentoré Amadou dans sa quête de maintes façons: il l'écoute attentivement lorsque ce dernier invente des histoires, il l'encourage (FBR, 50, 82, 83, 85, 86, 88, 91, 92, 124), lui offre son chapeau de Camargue $(F B R, 130,199)$ - chapeau dont il ne se départit pourtant jamais (FBR, 88, 197). Amadou lui en sait gré: "[J]e te remercie de m’avoir donné un coup de main. Ça m’a fait du bien.» (FBR, 93); «je me sens en paix» (FBR, 140).

49 Frédéric Raynaud, «Les gardians de Camargue et leurs chevaux, patrimoine archivistique et photographique pour une étude historique».

50 Jean-Pierre Lapointe, «Narcisse travesti. L'altérité des sexes chez trois romanciers québécois contemporains", p. 15. 
décline de deux façons dans Faites de beaux rêves: le preux chevalier et le chevalier errant. Par ses qualités de force et de vigueur, par ses valeurs humaines et spirituelles, Théo incarne la figure du noble paladin qui se porte à la défense des faibles (son frère, la Ceinture verte, Jane) et se met au service des dames, comme le donne à voir cet extrait:

Amadou entendit son frère embrasser plusieurs fois Limoilou. Il devait la serrer contre lui: elle avait un peu de mal à respirer.

- Tu devrais... faire un peu d'air, dit-elle.

- Je vais ouvrir un mousquetaire, dit Théo.

Il se leva en passant par-dessus Limoilou et Amadou, et il détacha le carreau de toile qui bouchait la fenêtre, près de l'entrée. Ensuite il sortit dehors.

- Pas de nuages, dit-il en rentrant. C'est plein d'étoiles, il va faire beau demain pour la course.

- Reviens te coucher dans le sac familial, espèce de fou, dit Limoilou. (FBR, 158;

l'auteur souligne)

On peut s'interroger sur l'effet de sens que produit le recours aux paronymes moustiquaire et mousquetaire. D'une part, on voit par ce jeu de mots que la carnavalisation du personnage transparaît jusque dans son langage. L'énoncé est porteur d'une disjonction où sont juxtaposées deux antinomies, l'une renvoyant à la «parole de tous ${ }^{51}$ » propre au réalisme familier, l'autre mettant à distance la pensée commune pour y substituer un intertexte qui ouvre l'esprit à un imaginaire sans limites, à des possibles sans fin. D'autre part, le terme «mousquetaire» fait écho à l'attitude chevaleresque de Théo. On le voit ici au chevet de sa belle, Limoilou. Il se montrera aussi galant en maintes autres occasions, trait que le preux chevalier partage avec le chevalier errant. Dans l'extrait suivant, Jacques Poulin convoque Don Quichotte, «création ambiguë de Cervantes ${ }^{52}$ », icône incontournable du patrimoine littéraire mondial:

Théo se leva et salua d'un grand coup de chapeau, à la manière de Don Quichotte s'inclinant devant sa Dulcinée du Toboso. La fille recula d'un pas.

- Excusez-moi, dit-elle. J'allais seulement chercher de l'eau.

- Vous êtes très jolie, dit Théo. Êtes-vous amoureuse de quelqu'un? (FBR, 71)

Deux thèmes s'entrecroisent dans cet énoncé hybride. D'un côté s'y révèlent tous les traits de l'amour courtois et de l'honneur chevaleresque, symbolisés par la présence de Dulcinée (Jane). De l'autre transparaît le sentiment du tragique que nous inspire Don Quichotte (Théo). S'il joue les grands séducteurs à la recherche de nouvelles conquêtes amoureuses, cela n'empêche pas Théo d'affirmer (aux dires de Jane) «qu'il est amoureux de Limoilou» (FBR, 181). Le versant négatif de l'amour, c'est bien sûr

51 Micheline Cambron, Une société, un récit. Discours culturel au Québec (1967-1976), p. 141.

52 «Don Quichotte [...] est à la fois la figure du chevalier héroïque dont l'idéalité transcende la contingence du monde factuel, le sympathique Alonso Quixana dont l'engouement pour les romans de chevalerie prête à sourire et une création ambiguë de Cervantes qui oscille entre la farce et la tragédie.» Vincent Jouve, L'effet-personnage dans le roman, Paris, Presses universitaires de France, coll. «Écriture», 2001, p. 66. 
l'échec de la quête amoureuse. Théo avait cette impression «qu'il avait une chance incroyable d'être en vie et d'être avec elle, et en même temps le sentiment que ça ne pouvait pas durer» $(F B R, 134)$. Dans un autre passage du roman, on apprend que c'est Théo, dans sa douce folie des grandeurs imaginaires, qui a donné le surnom «Limoilou» à leur amie d'enfance $(F B R, 143)$. Amadou explique l'origine du patronyme: "[ [] $\mathrm{l}$ y a un vieux manoir qui s'appelle Limoilou dans un petit village de la Bretagne, tout près de Saint-Malo.» (FBR, 143) Puis il fait cette révélation surprenante: «[C]'est dans ce manoir que Jacques Cartier est allé mourir.» (FBR, 144) Peut-on y voir une sorte de prémonition? Limoilou symbolise pour Théo une double quête inachevée, celle de l'amour et de l'imaginaire qui, finalement, se confondent, à l'instar de celle de Don Quichotte pour qui Dulcinée du Toboso demeurera à jamais insaisissable parce que fictive. L'acte donquichottesque n'est-il pas la quête d'un idéal inatteignable, néanmoins perpétuellement recherché? «Don Quichotte porte la littérature en lui comme une incurable blessure ${ }^{53}$ ", écrit Marthe Robert. Tout comme «le récit de Cervantes utilise les mythes littéraires et le discours du passé pour susciter un nouvel univers mythique ${ }^{54}$ ", les inventions farfelues et souvent dérisoires du Théo de Jacques Poulin ne seraient peut-être en définitive rien d'autre qu'une tentative toujours renouvelée de recréation de soi et du monde. De l'amour aussi.

\section{LE COWBOY}

Outre la figure du chevalier, que l'on a associé au type "héros légendaire» de la classification de Lapointe, se trouve celle du cowboy. Cet archétype semble tout à fait à sa place dans le contexte carnavalesque que l'on connaît. Au moment où le groupe se réunit pour se raconter des fables, notamment des "histoires du Far-West» (FBR, 140), Théo choisit d'incarner Buffalo Bill, ayant tous les attributs physiques et vestimentaires attendus pour le faire. Affublé d'un chapeau de cavalier, un «Smith \& Wesson » $(F B R, 135,172-173,199)$ à la hanche, ce grand gaillard à l'allure athlétique $(F B R, 111)$ porte « une barbe de trois jours [...] forte et très noire» $(F B R, 127)$. Quant à Jane, c'est en Calamity Jane qu'elle se déguise, empruntant à l'insu de son acolyte sa «veste de cuir doublée en mouton» $(F B R, 134)$ et son revolver. On assiste ici à deux transpositions littéraires de personnages historiques de l'époque de la conquête de l'Ouest. Dans Univers du western, Georges-Albert Astre et Albert-Patrick Hoarau décrivent le western très succinctement. Il s'agit en essence de l'aventure d'un héros se déroulant dans un paysage de l'Ouest. L'époque dont traite le western (18501900) correspond à la conquête d'un espace démesuré par des pionniers en marche vers l'ouest du continent, conquête civilisatrice qui se confond, dans l'imaginaire, avec celle d'un rêve fabuleux, l'American Dream : «[L]'Ouest symbolise la disponibilité, la nouvelle naissance de l'homme sur une terre heureuse et libre ${ }^{55}[\ldots]$.» Cette

53 Alain Rey (dir.), «Don Quichotte», Le Petit Robert des noms propres. Édition 2015, Paris, Le Robert, 2014, p. 669.

54 Ibid.

55 Georges-Albert Astre et Albert-Patrick Hoarau, Univers du western. Sources, structures, données permanentes, évolution, mythologie, Paris, Seghers, coll. «Cinéma Club», 1973, p. 21. 
donne historique a des conséquences sur le plan de la création artistique, comme en témoigne Jean Morency:

[L]'américanité évoque davantage une quête identitaire américaine qu'une affirmation de cette même identité qui se ferait ex nihilo. D'où l'importance du thème de la transformation et de la métamorphose dans plusieurs textes fondateurs des littératures d'Amérique, puisque l'américanité résulte nécessairement d'un phénomène de différenciation culturelle, voire anthropologique, qui s'inscrit entre deux états ou deux stades de civilisation ${ }^{56}$.

Ainsi, transposée textuellement dans le genre du western, cette aventure de la "quête identitaire américaine» donne lieu, selon Astre et Hoarau, à la forme narrative de l'itinéraire ${ }^{57}$, qui se construit par divers procédés: thème de la quête (qui peut parfois correspondre à une «errance infinie»), mise en scène de «l'accomplissement d'une promesse ou d'un engagement (tacite ou explicite) liant le héros au groupe» (par exemple, protéger un être vulnérable) et "résolution d'un antagonisme au profit des valeurs simples, naturelles de la solidarité humaine» (autrement dit, réconciliation momentanée de principes opposés: ordre/liberté, cowboy/ Indien, etc.). Dans Faites de beaux rêves, l'un des «beaux rêves» d'Adamou, justement, illustre bien tous les éléments de l'itinéraire narratif de la geste de l'Ouest dont parlent Astre et Hoarau:

- Mon frère Théo avait la vieille Winchester de Sitting Bull en travers des genoux [...]. [...] Il faisait claquer son fouet au-dessus des trois paires de chevaux qui tiraient notre chariot [...]. Théo me disait de ne pas regarder en arrière. Je me retournais quand même pour voir la caravane des émigrants qui nous suivaient dans leurs chariots depuis qu'on avait quitté Independance sur les bords du Missouri. Théo disait qu'on pouvait prendre la piste de l'Oregon ou celle de Santa Fe. Les villes s'appelaient Abilene, Fort Laramie et Dodge City. Théo n'arrêtait pas de faire claquer son fouet, il voulait aller plus vite que Kit Carson et William F. Cody. C'était nécessaire d'arriver au pied des Rocheuses avant les premières neiges d'octobre. Il se méfiait de Cochise et de Geronimo dans les prairies. Et il disait que si on allait plus au Sud, plus loin que le Grand Canyon du Colorado, on pouvait trouver l'Or de la Californie et la Terre Promise. (FBR, 140-141)

Sont réunis dans cet extrait les trois éléments de l'«itinéraire»: la quête à l'américaine (migration de Théo et d'Amadou vers l'Ouest), l'aide au prochain (Théo guide la caravane, instruit Amadou) et la synthèse des oppositions (aventurier solitaire/ groupe social, arrière/avant, passé/futur, cowboys/Indiens). En somme, ce rêve est porteur des linéaments d'une éventuelle conquête de l'Ouest américain par le jeune

56 Jean Morency, «Les modalités du décrochage européen des littératures américaines», Gérard Bouchard et Yvan Lamonde (dir.), Québécois et Américains. La culture québécoise aux XIX et XXe siècles, Montréal, Fides, 1995, p. 161-162; l'auteur souligne.

57 Georges-Albert Astre et Albert-Patrick Hoarau, Univers du western, p. 45-46. Les deux prochaines citations se trouvent aux mêmes pages. 
écrivain en devenir - qui se matérialisera dans Volkswagen Blues ${ }^{58}$ - et, conséquemment, de la transformation identitaire et culturelle de ce dernier.

Mais il y a plus encore. À la fonction identitaire de l'aventure westernienne se greffe une fonction éthique. Comme l'expliquent Astre et Hoarau, «l'affirmation du soi-même et de ses seules valeurs [...], c'est précisément l'attitude héroïque fondamentale; le héros "est un archétype, c'est-à-dire presque dieu, ou qu'il participe de la nature divine. Psychiquement le soi est une imago dei dont il ne peut être distingué empiriquement $[\ldots]^{\prime 59}$ ». Dans le récit (et particulièrement dans le rêve d'Amadou), Théo fait «figure de démiurge (ainsi que le suggère le nom) ${ }^{60}$ », précise François Gallays. Comme le héros du western, il incarne un idéal de pureté où «l'adéquation entre l'intérieur et l'extérieur est parfaite, l'équilibre entre l'homme et le monde pleinement assuré ${ }^{61}$ ». Par opposition à Amadou, être foncièrement divisé (commis/ écriture), Théo apparaît «organiquement et moralement homogène ${ }^{62}$ ». De ce trait de caractère distinctif d'un être en parfait accord avec lui-même résultent deux attitudes qui se rencontrent fréquemment chez le héros du western, soit l'authenticité et la franche jovialité, cette dernière prenant la forme d'un humour salé aux origines populaires (vulgarité, cynisme, rire, farces) ${ }^{63}$. Dans le roman qui nous occupe, on note que Théo n'hésite jamais à livrer le fond de sa pensée (FBR, 79, 127). Quant à son attitude bouffonne, elle s'illustre, entre autres, par son penchant à proférer des jurons (FBR, 17, 21, 24, 41, 48, 55, 58, 59, 60, 69, 76, 77, 81, 82, 88, 98, 108, 109, 113, 116, 131, 135, 167, 175, 179, 183 et 190!). Sans ses joyeux blasphèmes, Théo ne serait pas Théo, comme le souligne sans ironie Amadou: «- Tabarnak! entendit le commis [...]. - Je suis content d'entendre ça, dit le commis [...]. Je commençais à me demander si t'étais dans ton état normal. J'avais pas entendu de tabarnak depuis une heure ou deux. » (FBR, 131; l'auteur souligne) Afin d'excuser en quelque sorte ce trait de personnalité plutôt coloré, Amadou dit: «Mon frère Théo passe son temps à dire tabarnak et il engueule tout le monde pour rien. Ça veut rien dire $^{64 . »(F B R, ~ 195) ~}$

58 Jacques Poulin, Volkswagen Blues. Roman, Montréal/Arles, Leméac/Actes Sud, coll. «Babel», 1998 [1984], 323 p. Désormais, les références à cet ouvrage seront indiquées par le sigle $V B$ suivi du folio, et placées entre parenthèses dans le texte.

59 Georges-Albert Astre et Albert-Patrick Hoarau, Univers du western, p. 106; la citation est de Carl Jung (Métamorphoses de l'âme et ses symboles, 1952 [1912]); les auteurs soulignent.

60 François Gallays, «Faites de beaux rêves».

61 Bernard Dort (Le western, Paris, Union générale d'éditions, coll. 10/18) cité dans Georges-Albert Astre et Albert-Patrick Hoarau, Univers du western, p. 107.

62 Georges-Albert Astre et Albert-Patrick Hoarau, Univers du western, p. 108; les auteurs soulignent.

63 Ibid., p. 69.

64 Ici, on donnera tort à Amadou en disant que l'on peut certainement voir dans ce trait du personnage, ainsi que dans son goût prononcé pour l'alcool, l'influence des maîtres américains sur l'imaginaire de Jacques Poulin. Pierre Filion affirme que «[s]i l'on boit beaucoup [dans Faites de beaux rêves], c'est un peu en hommage à Hemingway, qui levait le coude plus souvent qu'à son tour» (Pierre Filion, «Vivre entre le rêve et la réalité» $[F B R, 5-6])$. Par ailleurs, l'usage abondant de jurons rapproche le personnage de Théo de celui de J. D. Salinger, Holden Caulfield (The Catcher in the Rye, 1951). L'admiration de Poulin pour Salinger est bien connue. En 1972, soit deux ans avant la publication de Faites de beaux rêves, il l'affirmait clairement: «L'histoire que j'aimerais écrire, ça ressemblerait au Vieil homme et la mer d'Hemingway, à cause de la simplicité, à L'écume des jours de Boris Vian à cause de la tendresse, à Nous autres, les Sanchez de Catherine Paysan, à cause de la chaleur humaine, et à L'attrape-cœur de Salinger pour les trois raisons que je viens de dire [...].» (Rachel Cloutier, Rodrigue Gignac, Vincent Nadeau et Richard Plamondon, «Entrevue avec 
Quoique solitaire de nature, l'aventurier ne chemine pas complètement seul. Parmi les protagonistes usuels du western, on trouve le hors-la-loi, le shérif, l'Indien et, bien sûr, la femme. Sans elle, c'est un lieu commun de l'affirmer, il ne saurait y avoir de western. Reste à voir en quoi sa présence est absolument requise. À ce sujet, Astre et Hoarau écrivent: «[L]a femme westernienne est en vérité nécessaire, moins pour créer du romanesque que pour mettre en valeur, finalement, le principe viril qui est son antagoniste ${ }^{65}$ [...].» Le genre serait-il antiféminin? À cela, les auteurs répondent qu'il s'agit plutôt d'une nécessité structurelle: si le western se veut la mise en scène d'une sorte d'homme «adamique», rien d'étonnant à ce qu'apparaisse à ses côtés «l'Ève primordiale ${ }^{66}$ »! Il est vrai que, dans ce genre où le protagoniste se caractérise souvent par une "masculinité essentielle ${ }^{67}$ », la femme semble être réduite à un «principe féminin» visant à faire tomber les hommes. C'est ce qu'on observe à tout le moins dans Faites de beaux rêves, où l'on voit la jeune «secrétaire médicale» $(F B R, 78)$ dans le rôle de Calamity Jane ${ }^{68}$, véritable «fille de saloon excitante ${ }^{69}$ » aux «longues jambes bronzées» $(F B R, 76)$ à la «Marlene Dietrich» $(F B R, 80)$, dont «les seins [...] se dessin[ent] sous la mince chemise» (FBR, 74), qui joue à l'effeuilleuse devant une foule soûle et gouailleuse (FBR, 149, 155). La «belle Calamity» $(F B R, 169)$, comme l'appelle Théo, est fort consciente de l'attrait sexuel qu'elle exerce sur la gent masculine - «Théo $[\ldots]$ a envie de moi à cause de mes jambes.» $(F B R, 100)$-, et le jeu de la séduction paraît être l'unique motivation de ce personnage typiquement westernien - «Il me regarde, dit-elle en souriant. J'ai toujours envie que les hommes me regardent.» $(F B R, 180)$ En définitive, et comme on pouvait s'y attendre en vertu du pacte de lecture que nous propose cette fiction à l'accent westernien, la présence de Jane dans l'histoire sert à mettre en valeur le héros qui aura à deux reprises (FBR, 149, 155) l'honneur (et le courage!) de se porter au secours de cette jolie femme fatale exposée à un gravissime danger: "- Tabarnak! dit Théo. Calamity fait un autre striptease! Faut que j'aille la sortir de là avec mon Smith \& Wesson!» (FBR, 155)

Il ne faut toutefois pas se bercer d'illusions. En fin renard, Jacques Poulin se joue ici du code romanesque comme Théo s'amuse (un peu à nos dépens) à jouer le

Jacques Poulin», Nord, n 2, hiver 1972, p. 10, cité dans Laurent Mailhot, «Présentation. Le voyage total», p. 3.) Faites de beaux rêves ne constitue-t-il pas la réalisation (fort réussie) d'un tel programme?

65 Georges-Albert Astre et Albert-Patrick Hoarau, Univers du western, p. 134-135.

66 Ibid., p. 135

67 Ibid., p. 108.

68 Martha Jane Cannaray, alias Calamity Jane (1852-1903), est un personnage de la conquête de l'Ouest américaine, auteure d'exploits remarquables dans le domaine équestre et le maniement des armes, qui lui firent une réputation sensationnelle de son vivant. Son histoire personnelle, cependant, est plutôt sombre (alcoolisme, déchéance, pauvreté). Comme plusieurs personnages historiques, soulignent Astre et Hoarau, «[l]es transpositions filmiques du personnage vont [...] toutes dans le sens d'une idéalisation étonnante, comme si, là encore, la "récupération" d'un personnage de hors-la-loi témoignait aujourd'hui des vertus d'aventure et d'héroïsme de la race éteinte de ces surhommes et de ces surfemmes qui "firent l'Amérique" » (Georges-Albert Astre et Albert-Patrick Hoarau, Univers du western, p. 136). La manière dont Jacques Poulin dépeint Calamity Jane dans Faites de beaux rêves ne correspond que très peu à l'image hollywoodienne de l'«amazone indomptable» (ibid., p. 137). Certes, Jane tente de se donner des airs de rebelle en agrémentant son costume western du Smith \& Wesson de Théo (FBR, 134-135). Néanmoins, ce sont ses traits de femme fatale qui prédominent, ainsi qu'un certain défaitisme coupable (FBR, 180, 191-192).

Georges-Albert Astre et Albert-Patrick Hoarau, Univers du western, p. 132 
justicier intrépide et macho. Il aurait été facile de tomber dans le piège du jugement de valeur, de ne voir dans Faites de beaux rêves que la réplique du schéma narratif très convenu (et sexiste, n'ayons pas peur des mots) du western dans sa facture la plus traditionnelle. Pour dégager la pleine signification de l'œuvre, il fallait plonger sous les apparences. Ainsi, par le biais de cette "construction hybride complexe ${ }^{70}$ " qu'est le personnage de Théo, l'auteur souligne, quoique très subtilement (mais là ne réside-t-elle pas en essence la force de son style?), les vertus d'une vie cohérente. Car il faut bien l'admettre: dans son rôle de Buffalo Bill, Théo est on ne peut plus fidèle à lui-même. Il est ce qu'il doit être, un héros conséquent, un demi-dieu en quelque sorte à l'image du petit-fils de Sisyphe, Bellérophon ${ }^{71}$, dirigeant sa monture Pégase avec fougue et agilité.

Mais pourquoi Théo a-t-il choisi très précisément d'incarner Buffalo Bill plutôt que Jesse James - dont il est fait mention dans Faites de beaux rêves $(F B R, 199)$ et dans Volkswagen Blues (VB, 155-156, 158) - ou tout autre personnage célèbre de l'épopée western (Daniel Boone, David Crockett, Kit Carson, etc.)? De son vrai nom William Frederick Cody (1846-1917), ce héros légendaire de la conquête de l'Ouest a pratiqué divers métiers: cow-boy, trappeur, chercheur d'or, cavalier pour le Pony Express, éclaireur chez les nordistes pendant la guerre de Sécession, membre de la cavalerie américaine en lutte contre les Indiens et chasseur de bisons. Fondateur du Wild West Show (1883), il produisit ce gigantesque cirque aux États-Unis et en Europe pendant une trentaine d'années. L'originalité de son entreprise tient au fait qu'elle se situe à «une époque-charnière ${ }^{72}$ », survenant précisément au moment où «l'Ouest sauvage est encore une réalité prestigieuse, mais l'aura qui l'environne s'affaiblit ${ }^{73}$ ». «Roi des hommes de la Frontière ${ }^{74} »$, W. F. Cody alias Buffalo Bill est à cheval entre deux mondes, l'ancien et le nouveau. De ce fait, il est sans contredit le personnage historique de l'entre-deux par excellence: cow-boy (gardien et conducteur de troupeau de bisons) et cowboy (acteur jouant la scène de la chasse aux bisons) évoluant entre le réel (les plaines américaines) et l'imaginaire (la plaine théâtralisée). Autrement dit, avec lui s'achève la grande geste de l'Ouest, qui est action conquérante, et débute le mythe de l'Ouest, souvenir de l'épopée colonisatrice à l'aube du

70 Mikhaïl Bakhtine cité dans André Belleau, «La dimension carnavalesque du roman québécois», p. 221.

71 Cherchant un jour à s'élever dans les cieux jusqu'à l'Olympe, Bellérophon fait une chute fatale et est condamné à errer en solitaire jusqu'à sa mort, ce qui n'est pas sans rappeler les affres du destin de son aïeul et, dans le cas qui nous occupe, la fin du parcours de Théo dans Volkswagen Blues. Son aventure ressemble à celle de l'homme absurde dont Albert Camus donne trois illustrations dans Le mythe de Sisyphe: «L'amant, le comédien ou l'aventurier jouent l'absurde. [...] Ils savent, voilà toute leur grandeur, et c'est en vain qu'on veut parler à leur propos de malheur caché ou des cendres de la désillusion. Être privé d'espoir, ce n'est pas désespérer. [...] Ils ne cherchent pas à être meilleurs, ils cherchent à être conséquents. Si le mot sage s'applique à l'homme qui vit de ce qu'il a, sans spéculer sur ce qu'il n'a pas, alors ceux-là sont des sages.» (Albert Camus, Le mythe de Sisyphe, Paris, Éditions Gallimard, 2005 [1942], coll. «Folio/Essais», p. 125-126.) En tant que chevalier, gardian et cowboy, le Théo de Jacques Poulin n'est-il pas à la fois l'amant, le comédien et l'aventurier dont parlait Camus? À n'en pas douter, il incarne de manière exemplaire l'homme absurde, lucide et conséquent, prince «sans royaume» conscient que «toutes les royautés sont illusoires» (Ibid., p. 125).

72 Georges-Albert Astre et Albert-Patrick Hoarau, Univers du western, p. 70.

73 Ibid.; les auteurs soulignent.

74 Ibid. 
devenir cinématographique. Dans Faites de beaux rêves, Théo se présente bel et bien sous les traits de l'homme de la frontière et du cirque. Accoutré d'un costume de cowboy, un pied dans le réel et l'autre dans l'imaginaire, il narre des récits, fabule et chante. De plus, à l'instar de son modèle westernien, il vit en nomade sur deux continents (Europe, Amérique), et sa vieille Gipsy - roulotte élevée au rang de personnage avec ses 41 occurrences $^{75}$ - ne manque pas de rappeler à la fois les chariots des pionniers de l'Ouest américain (FBR, 140-141) et les wagons de cirque, «small, travelling circuses which crisscrossed America in brightly coloured wagons throughout the nineteenth century ${ }^{76} »$.

\section{«... FAITES DE BEAUX RÊVES»}

En résumé, c'est par l'entremise de ses personnages hauts en couleur que Jacques Poulin fait entendre, au sein de cette étrange petite fable, une multitude de voix évoquant la possibilité d'une réinvention du sujet individuel et collectif à l'aune d'une culture repensée en fonction d'un recentrement identitaire (décrochage européen ${ }^{77}$, américanité assumée). De ce fait, l'auteur représente de manière exemplaire le «conflit des $\operatorname{codes}^{78}$ " propre à la période des années 1970, moment où le rapport à l'espace est déterminant. Partant de l'image symbolique de la piste de course, le roman nous invite à interroger diverses postures idéologiques, à remettre en question le récit hégémonique du discours culturel québécois. Doit-on opter pour l'inertie ou le mouvement? Le cas échéant, quelle direction prendre? Sur le circuit du MontTremblant, "dix mille chevaux lâchés d'un coup» (FBR, 187), les voitures s'élançant dans une euphorique et périlleuse cavalcade. Les chevaux-vapeur ne peuvent néanmoins rien accomplir sans une force directrice qui oriente adéquatement toute leur puissance. Cette force de direction, ce sont les pilotes qui la détiennent ${ }^{79}$. Ainsi en est-il de l'imaginaire d'Amadou, énergie créatrice prête à s'élancer à la conquête du langage. Impuissant à bien la gouverner, le commis aux écritures fait appel à son frère, dont les qualités de pilote sont attestées, comme il l'avait entrevu dans ce rêve où Théo est conducteur de «trois paires de chevaux» (sa «formule 3 »?). Le cheval, «par sa puissante musculature et son caractère fougueux, symbolise l'inconscient, la force du désir, l'énergie libidinale ${ }^{80}$ ». La maîtrise du cheval symbolise donc le contrôle de ces pulsions. Cavalier et conducteur d'attelage, Théo personnifie ce modèle héroïque alliant énergie pulsionnelle et contrôle de soi qui servira d'inspiration à

75 À cela s'ajoutent cinq mentions du mot «Gipsy» à titre de code lors de la grande opération d'observation de la course par les comparses (FBR, 174, 178, 182, 190).

76 Elaine Walker, Horse, London, Reaktion Books Ltd, coll. «Animal Series», 2008, p. 159. «Petits cirques ambulants qui parcouraient l'Amérique à bord de wagons colorés au dix-neuvième siècle» (nous traduisons).

77 Jean Morency, «Les modalités du décrochage européen des littératures américaines».

78 André Belleau, «Le conflit des codes dans l'institution littéraire québécoise», Surprendre les voix, Montréal, Boréal, 1986, p. 167-174.

79 «Limoilou demanda si Fangio était le meilleur pilote de tous les temps. - Trouve-moi un autre pilote qui a gagné cinq fois le Championnat du monde, répondit Théo. Il avait les jambes croches comme un vieux cow-boy [...], mais quand il prenait le volant...» (FBR, 155)

80 Corinne Morel, Dictionnaire des symboles, mythes et croyances, Paris, L'Archipel, 2005, p. 226. 
Amadou, comme nous le montre la suite de l'œuvre. Ce sera également grâce à son frère qu'Amadou en viendra à interroger la partie américaine de lui-même ${ }^{81}$, ce qui le conduira jusqu'à San Francisco dans Volkswagen Blues, roman qui poursuit en quelque sorte l'histoire amorcée dans Faites de beaux rêves.

Le titre de ce quatrième roman de Jacques Poulin est à la fois injonctif et programmatique. Il indique que le rêve, préfigurant l'action transformationnelle, annonce le devenir (artistique), le nouvel homme à naître (écrivain). Dans et par le songe, Amadou se construit petit à petit, non pas à l'image de son frère, mais en tant qu'être qui pourra éventuellement, comme Théo a su le faire, transcender tous les dualismes pour les mettre au service de l'art. Il n'y a pas de destin sans récit, suggère Micheline Cambron. Par conséquent, on ne peut se définir et se transformer qu'en se racontant ${ }^{82}$, d'où la nécessité, pour faire sens de nos aventures personnelles et collectives, d'être à l'écoute des fictions porteuses de nos rêves, rêves qui seront, pour une large part, les réalités de demain: «Si la littérature n’a pas pour finalité d'être efficace $[\ldots]$, elle peut sûrement [...] favoriser l'apparition et le nécessaire rapprochement d'un certain nombre de conditions d'ordre intellectuel et psychologique qui, elles, permettent et même hâtent les changements sociaux et politiques ${ }^{83}$.» Publié à mi-chemin entre les années 1960 et 1980, dans la foulée de nombreux récits de décolonisation ${ }^{84}$, Faites de beaux rêves annonce l'apparition prochaine en littérature québécoise d'univers romanesques postcoloniaux ${ }^{85}$ peuplés d'êtres réconciliés avec eux-mêmes ${ }^{86}$, dans un nouveau rapport à la culture et à l'espace.

81 L'expression est de Jacques Poulin lui-même: voir Jean Morency, «Les modalités du décrochage européen des littératures américaines», p. 170.

82 «L'identité [...] ne saurait avoir d'autre forme que narrative, car se définir c'est toujours ultimement se raconter. Une collectivité (ou un individu [...]) se définirait donc à travers les histoires qu'elle se raconte à elle-même sur elle-même [...].» Micheline Cambron, ibid., p. 176.

83 André Belleau, «Littérature et politique», Surprendre les voix, p. 75-76.

84 Si l'on en croit le titre de l'ouvrage de Maurice Arguin, la période du colonialisme se terminerait aux alentours de 1965: Maurice Arguin, Le roman québécois de 1944 à 1965. Symptômes du colonialisme et signes de libération, préface de Maurice Lemire, Montréal, L’Hexagone, coll. «Centre de recherche en littérature québécoise», 1989, 277 p.

85 Marie Vautier, «Les métarécits, le postmodernisme et le mythe postcolonial au Québec. Un point de vue de la "marge" », Études littéraires, vol. XXVII, n 1, été 1994, en ligne: http://id.erudit.org/iderudit/501067ar (page consultée le 8 novembre 2019).

86 «L'évolution des personnages au cours des romans successifs révèle comment ils réussissent progressivement à surmonter la dualité des oppositions binaires, en les combinant dans une fusion harmonieuse.» Jaap Lintvelt, Aspects de la narration. Thématique, idéologie et identité, p. 217-218. 